\title{
ON A DIFFERENTIAL EQUATION FOR KOORNWINDER'S GENERALIZED LAGUERRE POLYNOMIALS
}

\author{
J. KOEKOEK AND R. KOEKOEK
}

(Communicated by Kenneth R. Meyer)

ABSTRACT. Koornwinder's generalized Laguerre polynomials $\left\{L_{n}^{\alpha, N}(x)\right\}_{n=0}^{\infty}$ are orthogonal on the interval $[0, \infty)$ with respect to the weight function $\frac{1}{\Gamma(\alpha+1)} x^{\alpha} e^{-x}+N \delta(x), \alpha>-1, N \geq 0$. We show that these polynomials for $N>0$ satisfy a unique differential equation of the form

$$
N \sum_{i=0}^{\infty} a_{i}(x) y^{(i)}(x)+x y^{\prime \prime}(x)+(\alpha+1-x) y^{\prime}(x)+n y(x)=0,
$$

where $\left\{a_{i}(x)\right\}_{i=0}^{\infty}$ are continuous functions on the real line and $\left\{a_{i}(x)\right\}_{i=1}^{\infty}$ are independent of the degree $n$. If $N>0$, only in the case of nonnegative integer values of $\alpha$ this differential equation is of finite order.

\section{INTRODUCTION}

In 1984, T. H. Koornwinder found the polynomials which are orthogonal with respect to a weight function consisting of the classical Jacobi weight function together with delta functions at the endpoints of the interval of orthogonality $[-1,1]$. (See [2].) As a limiting case, he found the generalized Laguerre polynomials which are orthogonal on the interval $[0, \infty)$ with respect to the weight function $\frac{1}{\Gamma(\alpha+1)} x^{\alpha} e^{-x}+N \delta(x), \alpha>-1$ and $N \geq 0$. In this paper we consider only Koornwinder's generalized Laguerre polynomials $\left\{L_{n}^{\alpha, N}(x)\right\}_{n=0}^{\infty}$ defined by

$$
L_{n}^{\alpha, N}(x)=\left[1+N\left(\begin{array}{c}
n+\alpha \\
n-1
\end{array}\right)\right] L_{n}^{(\alpha)}(x)+N\left(\begin{array}{c}
n+\alpha \\
n
\end{array}\right) \frac{d}{d x} L_{n}^{(\alpha)}(x),
$$

where $L_{n}^{(\alpha)}(x)$ denotes the classical Laguerre polynomial given by

$$
L_{n}^{(\alpha)}(x)=\left(\begin{array}{c}
n+\alpha \\
n
\end{array}\right) \sum_{k=0}^{n} \frac{(-n)_{k}}{(\alpha+1)_{k}} \frac{x^{k}}{k !}, \quad n=0,1,2, \ldots
$$

For details the reader is referred to $[2,1]$. Note that $L_{n}^{\alpha, 0}(x)=L_{n}^{(\alpha)}(x)$.

Received by the editors February 17, 1990.

1980 Mathematics Subject Classification (1985 Revision). Primary 33A65. 
For the polynomials $\left\{L_{n}^{\alpha, N}(x)\right\}_{n=0}^{\infty}$, a differential equation of the form

$$
N \sum_{i=0}^{\infty} a_{i}(x) y^{(i)}(x)+x y^{\prime \prime}(x)+(\alpha+1-x) y^{\prime}(x)+n y(x)=0,
$$

where $\left\{a_{i}(x)\right\}_{i=0}^{\infty}$ are continuous functions on the real line and $\left\{a_{i}(x)\right\}_{i=1}^{\infty}$ are independent of $n$, was found only for special nonnegative integer values of $\alpha$.

For $\alpha=0, \alpha=1$, and $\alpha=2$, H. L. Krall, A. M. Krall, and L. L. Littlejohn obtained the following differential equations:

$$
\begin{aligned}
& -\frac{1}{2} N x^{2} y^{(4)}(x)+N\left(x^{2}-2 x\right) y^{(3)}(x)+\left[-\frac{1}{2} N x^{2}+(3 N+1) x\right] y^{\prime \prime}(x) \\
& +[1-(N+1) x] y^{\prime}(x)+n\left[\frac{1}{2}(n+1) N+1\right] y(x)=0
\end{aligned}
$$

$$
\begin{gathered}
\frac{1}{6} N x^{3} y^{(6)}(x)+N\left(-\frac{1}{2} x^{3}+2 x^{2}\right) y^{(5)}(x)+N\left(\frac{1}{2} x^{3}-5 x^{2}+5 x\right) y^{(4)}(x) \\
+N\left(-\frac{1}{6} x^{3}+4 x^{2}-10 x\right) y^{(3)}(x)+\left[-N x^{2}+(6 N+1) x\right] y^{\prime \prime}(x) \\
+[2-(N+1) x] y^{\prime}(x)+n\left[\frac{1}{6}(n+1)(n+2) N+1\right] y(x)=0
\end{gathered}
$$

and

$$
\begin{aligned}
& -\frac{1}{24} N x^{4} y^{(8)}(x)+N\left(\frac{1}{6} x^{4}-x^{3}\right) y^{(7)}(x)+N\left(-\frac{1}{4} x^{4}+\frac{7}{2} x^{3}-7 x^{2}\right) y^{(6)}(x) \\
& \quad+N\left(\frac{1}{6} x^{4}-\frac{9}{2} x^{3}+21 x^{2}-14 x\right) y^{(5)}(x) \\
& +N\left(-\frac{1}{24} x^{4}+\frac{5}{2} x^{3}-\frac{45}{2} x^{2}+35 x\right) y^{(4)}(x) \\
& \quad+N\left(-\frac{1}{2} x^{3}+10 x^{2}-30 x\right) y^{(3)}(x)+\left[-\frac{3}{2} N x^{2}+(10 N+1) x\right] y^{\prime \prime}(x) \\
& \quad+[3-(N+1) x] y^{\prime}(x)+n\left[\frac{1}{24}(n+1)(n+2)(n+3) N+1\right] y(x)=0,
\end{aligned}
$$

respectively, in a different notation. See [3-9]. Note that in these cases $\left\{a_{i}(x)\right\}_{i=0}^{\infty}$ are polynomials and the differential equation is of order $2 \alpha+4$.

In this paper we give a differential equation of the form (3) for Koornwinder's generalized Laguerre polynomials $\left\{L_{n}^{\alpha, N}(x)\right\}_{n=0}^{\infty}$ for all $\alpha>-1$. It will appear that $\left\{a_{i}(x)\right\}_{i=0}^{\infty}$ are polynomials for all $\alpha>-1$, and that the differential equation is of order $2 \alpha+4$ for all nonnegative integer values of $\alpha$ if $N>0$. 


\section{THE DIFFERENTIAL EQUATION}

Consider the differential equation (3), where $\left\{a_{i}(x)\right\}_{i=0}^{\infty}$ are continuous functions on the real line, $a_{0}(x):=a_{0}(n, \alpha, x)$ and $a_{i}(x):=a_{i}(\alpha, x)$ for $i \geq 1$.

We will try to define the functions $\left\{a_{i}(x)\right\}_{i=0}^{\infty}$ in such a way that the polynomials $\left\{L_{n}^{\alpha, N}(x)\right\}_{n=0}^{\infty}$ satisfy a differential equation of the form (3) for all $\alpha>-1$ and $N \geq 0$.

Recall that the classical Laguerre polynomials $\left\{L_{n}^{(\alpha)}(x)\right\}_{n=0}^{\infty}$ satisfy the Laguerre equation given by

$$
x y^{\prime \prime}(x)+(\alpha+1-x) y^{\prime}(x)+n y(x)=0 .
$$

We set $y(x)=L_{n}^{\alpha, N}(x)$ in the differential equation (3) and use the definition (1) and the Laguerre equation (7) to find

$$
\begin{aligned}
& N\left[1+N\left(\begin{array}{c}
n+\alpha \\
n-1
\end{array}\right)\right] \sum_{i=0}^{\infty} a_{i}(x) D^{i} L_{n}^{(\alpha)}(x)+N^{2}\left(\begin{array}{c}
n+\alpha \\
n
\end{array}\right) \sum_{i=0}^{\infty} a_{i}(x) D^{i+1} L_{n}^{(\alpha)}(x) \\
& \quad+N\left(\begin{array}{c}
n+\alpha \\
n
\end{array}\right)\left[x \frac{d^{3}}{d x^{3}} L_{n}^{(\alpha)}(x)+(\alpha+1-x) \frac{d^{2}}{d x^{2}} L_{n}^{(\alpha)}(x)+n \frac{d}{d x} L_{n}^{(\alpha)}(x)\right]=0 .
\end{aligned}
$$

Differentiation of the Laguerre equation (7) leads to

$$
x \frac{d^{3}}{d x^{3}} L_{n}^{(\alpha)}(x)+(\alpha+2-x) \frac{d^{2}}{d x^{2}} L_{n}^{(\alpha)}(x)+(n-1) \frac{d}{d x} L_{n}^{(\alpha)}(x)=0 .
$$

Hence

$$
\begin{aligned}
& N\left[\sum_{i=0}^{\infty} a_{i}(x) D^{i} L_{n}^{(\alpha)}(x)+\left(\begin{array}{c}
n+\alpha \\
n
\end{array}\right) \frac{d}{d x} L_{n}^{(\alpha)}(x)-\left(\begin{array}{c}
n+\alpha \\
n
\end{array}\right) \frac{d^{2}}{d x^{2}} L_{n}^{(\alpha)}(x)\right] \\
& +N^{2}\left[\left(\begin{array}{c}
n+\alpha \\
n-1
\end{array}\right) \sum_{i=0}^{\infty} a_{i}(x) D^{i} L_{n}^{(\alpha)}(x)+\left(\begin{array}{c}
n+\alpha \\
n
\end{array}\right) \sum_{i=0}^{\infty} a_{i}(x) D^{i+1} L_{n}^{(\alpha)}(x)\right]=0
\end{aligned}
$$

for all real $x, \alpha>-1, N \geq 0$ and $n=0,1,2, \ldots$. Since the expressions between square brackets are independent of $N$ this implies that

$$
\sum_{i=0}^{\infty} a_{i}(x) D^{i} L_{n}^{(\alpha)}(x)+\left(\begin{array}{c}
n+\alpha \\
n
\end{array}\right) \frac{d}{d x} L_{n}^{(\alpha)}(x)-\left(\begin{array}{c}
n+\alpha \\
n
\end{array}\right) \frac{d^{2}}{d x^{2}} L_{n}^{(\alpha)}(x)=0
$$

and

$$
n \sum_{i=0}^{\infty} a_{i}(x) D^{i} L_{n}^{(\alpha)}(x)+(\alpha+1) \sum_{i=0}^{\infty} a_{i}(x) D^{i+1} L_{n}^{(\alpha)}(x)=0
$$

for all real $x$ and $n=0,1,2, \ldots$.

First of all we show that (8) and (9) have at most one solution for $\left\{a_{i}(x)\right\}_{i=0}^{\infty}$. This means that we have to show that

$$
\sum_{i=0}^{\infty} a_{i}(x) D^{i} L_{n}^{(\alpha)}(x)=0, \quad n=0,1,2, \ldots,
$$


$n \sum_{i=0}^{\infty} a_{i}(x) D^{i} L_{n}^{(\alpha)}(x)+(\alpha+1) \sum_{i=0}^{\infty} a_{i}(x) D^{i+1} L_{n}^{(\alpha)}(x)=0, \quad n=0,1,2, \ldots$,

rave only the trivial solution. Note that (10) and (11) imply, for all real $x$, that

$$
a_{0}(n, \alpha, x) L_{n}^{(\alpha)}(x)+\sum_{i=1}^{\infty} a_{i}(\alpha, x) D^{i} L_{n}^{(\alpha)}(x)=0, \quad n=0,1,2, \ldots,
$$

and

$$
a_{0}(n, \alpha, x) \frac{d}{d x} L_{n}^{(\alpha)}(x)+\sum_{i=1}^{\infty} a_{i}(\alpha, x) D^{i+1} L_{n}^{(\alpha)}(x)=0, \quad n=0,1,2, \ldots
$$

Substitution of $n=0$ and $n=1$ in (12) and (13) gives us

$$
a_{0}(0, \alpha, x)=0, \quad a_{0}(1, \alpha, x)=0, \quad \text { and } a_{1}(\alpha, x)=0
$$

for all real $x$. Now we set $n=2$ in (13) to obtain

$$
a_{0}(2, \alpha, x)=0 \text { for all real } x \text { except possibly for } x=\alpha+2,
$$

being the zero of $\frac{d}{d x} L_{2}^{(\alpha)}(x)$. Now we use the continuity of $a_{0}(x)$ to conclude that $a_{0}(2, \alpha, x)=0$ for all real $x$. Then, by setting $n=2$, we obtain from (12)

$$
a_{2}(\alpha, x)=0 \text { for all real } x .
$$

Repeating this process, we finally find

$$
\left\{\begin{array}{lll}
a_{0}(x)=a_{0}(n, \alpha, x)=0 & \text { for all real } x \text { and } n=0,1,2, \ldots \\
a_{i}(x)=a_{i}(\alpha, x)=0 & \text { for all real } x \text { and } i=1,2,3, \ldots
\end{array}\right.
$$

This proves that (10) and (11) have only the trivial solution. Hence (8) and (9) have at most one solution.

As in (4), (5), and (6) we try to find polynomial solutions for the functions $\left\{a_{i}(x)\right\}_{i=0}^{\infty}$. We may expect that

$$
a_{0}(x)=\left(\begin{array}{c}
n+\alpha+1 \\
n-1
\end{array}\right)
$$

Since the functions $\left\{a_{i}(x)\right\}_{i=1}^{\infty}$ are not allowed to depend on $n$, we can compute some of them by substituting small values of $n$ in (8). We find

$$
\begin{aligned}
a_{1}(x)= & -x \\
a_{2}(x)= & -\frac{1}{2}(\alpha+1) x^{2}+\frac{1}{2}(\alpha+2)(\alpha+3) x \\
a_{3}(x)= & -\frac{1}{12} \alpha(\alpha+1) x^{3}+\frac{1}{6}(\alpha+1)(\alpha+2)(\alpha+3) x^{2} \\
& -\frac{1}{12}(\alpha+1)(\alpha+2)(\alpha+3)(\alpha+4) x
\end{aligned}
$$


So we may guess that

$$
a_{i}(x)=\frac{1}{i !} \sum_{j=1}^{i}(-1)^{i+j+1}\left(\begin{array}{c}
\alpha+1 \\
j-1
\end{array}\right)\left(\begin{array}{c}
\alpha+2 \\
i-j
\end{array}\right)(\alpha+3)_{i-j} x^{j}, \quad i=1,2,3, \ldots
$$

We will prove that the polynomials $\left\{L_{n}^{\alpha, N}(x)\right\}_{n=0}^{\infty}$ satisfy the differential equation given by (3), (14), and (15).

\section{Proof of THE Differential equation}

We have to show that the polynomials $\left\{a_{i}(x)\right\}_{i=0}^{\infty}$ defined by (14) and (15) satisfy (8) and (9). We start with

$$
\begin{aligned}
& \sum_{i=1}^{\infty} a_{i}(x) D^{i} L_{n}^{(\alpha)}(x) \\
& \quad=\sum_{i=1}^{\infty} \sum_{j=1}^{i} \frac{(-1)^{i+j+1}}{i !}\left(\begin{array}{c}
\alpha+1 \\
j-1
\end{array}\right)\left(\begin{array}{c}
\alpha+2 \\
i-j
\end{array}\right)(\alpha+3)_{i-j} x^{j} D^{i} L_{n}^{(\alpha)}(x)
\end{aligned}
$$

where $x$ is real and $n$ is a nonnegative integer. Changing the order of summation twice, we obtain

$$
\begin{aligned}
& \sum_{i=1}^{\infty} a_{i}(x) D^{i} L_{n}^{(\alpha)}(x) \\
& \quad=\sum_{j=1}^{\infty}(-1)^{j+1}\left(\begin{array}{c}
\alpha+1 \\
j-1
\end{array}\right) x^{j} \sum_{i=j}^{\infty} \frac{(-1)^{i}}{i !}\left(\begin{array}{c}
\alpha+2 \\
i-j
\end{array}\right)(\alpha+3)_{i-j} D^{i} L_{n}^{(\alpha)}(x) \\
& \quad=\sum_{j=1}^{\infty}(-1)^{j+1}\left(\begin{array}{c}
\alpha+1 \\
j-1
\end{array}\right) x^{j} \sum_{i=0}^{\infty} \frac{(-1)^{i+j}}{(i+j) !}\left(\begin{array}{c}
\alpha+2 \\
i
\end{array}\right)(\alpha+3)_{i} D^{i+j} L_{n}^{(\alpha)}(x) \\
& =-\sum_{i=0}^{\infty}(-1)^{i}\left(\begin{array}{c}
\alpha+2 \\
i
\end{array}\right)(\alpha+3)_{i} \sum_{j=1}^{\infty}\left(\begin{array}{c}
\alpha+1 \\
j-1
\end{array}\right) \frac{x^{j}}{(i+j) !} D^{i+j} L_{n}^{(\alpha)}(x) .
\end{aligned}
$$

Now we use the definition of the classical Laguerre polynomials (2) to find

$$
\begin{aligned}
& \sum_{j=1}^{\infty}\left(\begin{array}{c}
\alpha+1 \\
j-1
\end{array}\right) \frac{x^{j}}{(i+j) !} D^{i+j} L_{n}^{(\alpha)}(x) \\
& \quad=\left(\begin{array}{c}
n+\alpha \\
n
\end{array}\right) \sum_{j=1}^{\infty} \sum_{k=0}^{\infty}\left(\begin{array}{c}
\alpha+1 \\
j-1
\end{array}\right) \frac{(-n)_{i+j+k} x^{j+k}}{(\alpha+1)_{i+j+k}(i+j) ! k !} \\
& \quad=\left(\begin{array}{c}
n+\alpha \\
n
\end{array}\right) \sum_{m=1}^{\infty} C_{m} x^{m}, \quad i=0,1,2, \ldots,
\end{aligned}
$$


where

$$
\begin{aligned}
C_{m} & =\sum_{j=1}^{m}\left(\begin{array}{c}
\alpha+1 \\
j-1
\end{array}\right) \frac{(-n)_{i+m}}{(\alpha+1)_{i+m}(i+j) !(m-j) !} \\
& =\frac{(-n)_{i+m}}{(\alpha+1)_{i+m}} \sum_{j=0}^{m-1}\left(\begin{array}{c}
\alpha+1 \\
j
\end{array}\right) \frac{1}{(i+j+1) !(m-1-j) !}, \quad m=1,2,3, \ldots
\end{aligned}
$$

Since $(i+j+1) !=(i+1) !(i+2)_{j}$,

$$
\left(\begin{array}{c}
\alpha+1 \\
j
\end{array}\right)=(-1)^{j} \frac{(-\alpha-1)_{j}}{j !} \text { and } \frac{1}{(m-1-j) !}=(-1)^{j} \frac{(-m+1)_{j}}{(m-1) !}
$$

we obtain

$$
\begin{aligned}
C_{m}=\frac{(-n)_{i+m}}{(\alpha+1)_{i+m}} \frac{1}{(m-1) !(i+1) !}{ }_{2} F_{1}\left(\begin{array}{r}
-m+1,-\alpha-1 \\
i+2
\end{array}\right. & ; 1), \\
& m=1,2,3, \ldots .
\end{aligned}
$$

Now we use the summation formula

$$
{ }_{2} F_{1}\left(\begin{array}{c}
a, b \\
c
\end{array} ; 1\right)=\frac{\Gamma(c-a-b) \Gamma(c)}{\Gamma(c-a) \Gamma(c-b)}, \quad c-a-b>0, c \neq 0,-1,-2, \ldots,
$$

to find for $m=1,2,3, \ldots$,

$$
\begin{aligned}
C_{m} & =\frac{(-n)_{i+m}}{(\alpha+1)_{i+m}} \frac{1}{(m-1) !(i+1) !} \frac{\Gamma(m+\alpha+i+2) \Gamma(i+2)}{\Gamma(m+i+1) \Gamma(\alpha+i+3)} \\
& =\frac{(-n)_{i+m}(\alpha+i+3)_{m-1}}{(\alpha+1)_{i+m}(m-1) !(i+m) !} .
\end{aligned}
$$

Hence, with (16) and (17) we have

$$
\begin{aligned}
& \sum_{i=1}^{\infty} a_{i}(x) D^{i} L_{n}^{(\alpha)}(x) \\
& \quad=-\left(\begin{array}{c}
n+\alpha \\
n
\end{array}\right) \sum_{i=0}^{\infty}(-1)^{i}\left(\begin{array}{c}
\alpha+2 \\
i
\end{array}\right)(\alpha+3)_{i} \sum_{m=1}^{\infty} \frac{(-n)_{i+m}}{(\alpha+1)_{i+m}} \frac{(\alpha+i+3)_{m-1}}{(m-1) !(i+m) !} x^{m} \\
& \quad=-\left(\begin{array}{c}
n+\alpha \\
n
\end{array}\right) \sum_{m=1}^{\infty} \sum_{i=0}^{\infty} \frac{(-\alpha-2)_{i}(-n)_{i+m}(\alpha+3)_{i+m-1}}{i !(\alpha+1)_{i+m}(m-1) !(i+m) !} x^{m} .
\end{aligned}
$$


Now we use the facts that $(-n)_{i+m}=(-n)_{m}(-n+m)_{i},(i+m) !=m !(m+1)_{i}$, and

$$
\frac{(\alpha+3)_{i+m-1}}{(\alpha+1)_{i+m}}=\frac{\alpha+i+m+1}{(\alpha+1)(\alpha+2)}
$$

to find

$$
\begin{aligned}
\sum_{i=1}^{\infty} a_{i}(x) D^{i} L_{n}^{(\alpha)}(x) \\
\quad=-\frac{\left(\begin{array}{c}
n+\alpha \\
n
\end{array}\right)}{(\alpha+1)(\alpha+2)} \sum_{m=1}^{\infty} \frac{(-n)_{m}}{(m-1) !} \frac{x^{m}}{m !} \sum_{i=0}^{\infty} \frac{(-\alpha-2)_{i}(-n+m)_{i}}{(m+1)_{i} i !}(\alpha+i+m+1)
\end{aligned}
$$

We split the last sum into two parts and use the summation formula (18), obtaining

$$
\begin{aligned}
& \sum_{i=0}^{\infty} \frac{(-\alpha-2)_{i}(-n+m)_{i}}{(m+1)_{i} i !}(\alpha+i+m+1) \\
& =(\alpha+m+1)_{2} F_{1}\left(\begin{array}{c}
-\alpha-2,-n+m ; 1 \\
m+1
\end{array}\right) \\
& +\frac{(\alpha+2)(n-m)}{(m+1)}{ }_{2} F_{1}\left(\begin{array}{c}
-\alpha-1,-n+m+1 ; 1 \\
m+2
\end{array}\right) \\
& =(\alpha+m+1) \frac{\Gamma(n+\alpha+3) \Gamma(m+1)}{\Gamma(m+\alpha+3) \Gamma(n+1)} \\
& +\frac{(\alpha+2)(n-m)}{(m+1)} \frac{\Gamma(n+\alpha+2) \Gamma(m+2)}{\Gamma(m+\alpha+3) \Gamma(n+1)} \\
& =(\alpha+1)\left(\begin{array}{c}
n+\alpha+1 \\
n
\end{array}\right) \frac{m !}{(\alpha+1)_{m+2}}[(m+2 \alpha+3) n+(\alpha+1)(\alpha+2)] \text {. }
\end{aligned}
$$

Hence

$$
\begin{aligned}
\sum_{i=1}^{\infty} a_{i}(x) D^{i} L_{n}^{(\alpha)}(x) \\
=-\frac{1}{(\alpha+2)}\left(\begin{array}{c}
n+\alpha \\
n
\end{array}\right)\left(\begin{array}{c}
n+\alpha+1 \\
n
\end{array}\right) \\
\quad \times \sum_{m=1}^{\infty} \frac{(-n)_{m}}{(\alpha+1)_{m+2}}[(m+2 a+3) n+(\alpha+1)(\alpha+2)] \frac{x^{m}}{(m-1) !}
\end{aligned}
$$

for all real $x$ and $n$ a nonnegative integer. 
To complete the proof of (8), we use (14) and (2) to find (20)

$$
\begin{aligned}
& a_{0}(x) L_{n}^{(\alpha)}(x)+\left(\begin{array}{c}
n+\alpha \\
n
\end{array}\right) \frac{d}{d x} L_{n}^{(\alpha)}(x)-\left(\begin{array}{c}
n+\alpha \\
n
\end{array}\right) \frac{d^{2}}{d x^{2}} L_{n}^{(\alpha)}(x) \\
& =\left(\begin{array}{c}
n+\alpha \\
n
\end{array}\right) \sum_{k=0}^{\infty}\left[\left(\begin{array}{c}
n+\alpha+1 \\
n-1
\end{array}\right) \frac{(-n)_{k}}{(\alpha+1)_{k}}+\left(\begin{array}{c}
n+\alpha \\
n
\end{array}\right) \frac{(-n)_{k+1}}{(\alpha+1)_{k+1}}\right. \\
& \left.=\left(\begin{array}{c}
n+\alpha \\
n
\end{array}\right) \sum_{k=0}^{\infty}\left[\begin{array}{c}
n+\alpha \\
n
\end{array}\right) \frac{(-n)_{k+2}}{(\alpha+1)_{k+2}}\right] \frac{x^{k}}{k !} \\
& \left.\quad+\left(\begin{array}{c}
n+\alpha+1 \\
n-1
\end{array}\right) \frac{(-n)_{k}}{(\alpha+1)_{k}}\left(\begin{array}{c}
n+\alpha \\
n
\end{array}\right) \frac{(-n)_{k+1}}{(\alpha+1)_{k+2}}\right] \frac{x^{k}}{k !} \\
& =\frac{1}{(\alpha+2)}\left(\begin{array}{c}
n+\alpha \\
n
\end{array}\right)\left(\begin{array}{c}
n+\alpha+1 \\
n
\end{array}\right) \\
& \times \sum_{k=1}^{\infty} \frac{(-n)_{k}}{(\alpha+1)_{k+2}}[n(k+2 \alpha+3)+(\alpha+1)(\alpha+2)] \frac{x^{k}}{(k-1) !}
\end{aligned}
$$

for all real $x$ and $n$ a nonnegative integer. With (19) and (20) we have proved (8). To show (9), we observe that (compare with (16))

$$
\begin{aligned}
& \sum_{i=1}^{\infty} a_{i}(x) D^{i+1} L_{n}^{(\alpha)}(x) \\
& \quad=-\sum_{i=0}^{\infty}(-1)^{i}\left(\begin{array}{c}
\alpha+2 \\
i
\end{array}\right)(\alpha+3)_{i} \sum_{j=1}^{\infty}\left(\begin{array}{c}
\alpha+1 \\
j-1
\end{array}\right) \frac{x^{j}}{(i+j) !} D^{i+j+1} L_{n}^{(\alpha)}(x)
\end{aligned}
$$

for all real $x$ and $n$ a nonnegative integer. In the same way as before, we find

$$
\begin{aligned}
\sum_{j=1}^{\infty} & \left(\begin{array}{c}
\alpha+1 \\
j-1
\end{array}\right) \frac{x^{j}}{(i+j) !} D^{i+j+1} L_{n}^{(\alpha)}(x) \\
& =\left(\begin{array}{c}
n+\alpha \\
n
\end{array}\right) \sum_{m=1}^{\infty} D_{m} x^{m}, \quad i=0,1,2, \ldots,
\end{aligned}
$$

where, for $m=1,2,3, \ldots$,

$$
\begin{aligned}
D_{m} & =\sum_{j=1}^{m}\left(\begin{array}{c}
\alpha+1 \\
j-1
\end{array}\right) \frac{(-n)_{i+m+1}}{(\alpha+1)_{i+m+1}(i+j) !(m-j) !} \\
& =\frac{(-n)_{i+m+1}(\alpha+i+3)_{m-1}}{(\alpha+1)_{i+m+1}(m-1) !(i+m) !} .
\end{aligned}
$$


Hence

$$
\begin{aligned}
\sum_{i=1}^{\infty} a_{i}(x) D^{i+1} L_{n}^{(\alpha)}(x) & \\
& =-\left(\begin{array}{c}
n+\alpha \\
n
\end{array}\right) \sum_{m=1}^{\infty} \sum_{i=0}^{\infty} \frac{(-\alpha-2)_{i}(-n)_{i+m+1}(\alpha+3)_{i+m-1}}{i !(\alpha+1)_{i+m+1}(m-1) !(i+m) !} x^{m} \\
& =-\frac{\left(\begin{array}{c}
n+\alpha \\
n
\end{array}\right)}{(\alpha+1)(\alpha+2)} \sum_{m=1}^{\infty} \frac{(-n)_{m+1}}{(m-1) !} \frac{x^{m}}{m !}{ }_{2} F_{1}\left(\begin{array}{c}
-\alpha-2,-n+m+1 ; 1 \\
m+1
\end{array}\right) \\
& =-\frac{\left(\begin{array}{c}
n+\alpha \\
n
\end{array}\right)}{(\alpha+1)(\alpha+2)} \sum_{m=1}^{\infty} \frac{(-n)_{m+1}}{(m-1) !} \frac{x^{m}}{m !} \frac{\Gamma(n+\alpha+2) \Gamma(m+1)}{\Gamma(m+\alpha+3) \Gamma(n)} \\
& =-\left(\begin{array}{c}
n+\alpha \\
n
\end{array}\right)\left(\begin{array}{c}
n+\alpha+1 \\
n-1
\end{array}\right) \sum_{m=1}^{\infty} \frac{(-n)_{m+1}}{(\alpha+1)_{m+2}} \frac{x^{m}}{(m-1) !} .
\end{aligned}
$$

With (19) and (21), we have found that

$$
\begin{aligned}
n \sum_{i=1}^{\infty} & a_{i}(x) D^{i} L_{n}^{(\alpha)}(x)+(\alpha+1) \sum_{i=1}^{\infty} a_{i}(x) D^{i+1} L_{n}^{(\alpha)}(x) \\
= & -\left(\begin{array}{c}
n+\alpha \\
n
\end{array}\right)\left(\begin{array}{c}
n+\alpha+1 \\
n-1
\end{array}\right) \\
& \times \sum_{m=1}^{\infty} \frac{(-n)_{m}}{(\alpha+1)_{m+2}}[(m+2 \alpha+3) n+(\alpha+1)(\alpha+2) \\
& +(\alpha+1)(m-n)] \frac{x^{m}}{(m-1) !} \\
= & -(n+\alpha+1)\left(\begin{array}{c}
n+\alpha \\
n
\end{array}\right)\left(\begin{array}{c}
n+\alpha+1 \\
n-1
\end{array}\right) \sum_{m=1}^{\infty} \frac{(-n)_{m}}{(\alpha+1)_{m+1}} \frac{x^{m}}{(m-1) !}
\end{aligned}
$$

for all real $x$ and $n$ a nonnegative integer. To complete the proof of (9), we use (14) and (2) to see that for all real $x$ and $n$ a nonnegative integer

$$
\begin{aligned}
& n a_{0}(x) L_{n}^{(\alpha)}(x)+(\alpha+1) a_{0}(x) \frac{d}{d x} L_{n}^{(\alpha)}(x) \\
& \quad=\left(\begin{array}{c}
n+\alpha \\
n
\end{array}\right)\left(\begin{array}{c}
n+\alpha+1 \\
n-1
\end{array}\right) \sum_{k=0}^{\infty} \frac{(-n)_{k}}{(\alpha+1)_{k+1}}[n(\alpha+k+1)+(-n+k)(\alpha+1)] \frac{x^{k}}{k !} \\
& =(n+\alpha+1)\left(\begin{array}{c}
n+\alpha \\
n
\end{array}\right)\left(\begin{array}{c}
n+\alpha+1 \\
n-1
\end{array}\right) \sum_{k=1}^{\infty} \frac{(-n)_{k}}{(\alpha+1)_{k+1}} \frac{x^{k}}{(k-1) !} .
\end{aligned}
$$

With (22) and (23) we have proved (9).

This shows that Koornwinder's generalized Laguerre polynomials $\left\{L_{n}^{\alpha, N}(x)\right\}_{n=0}^{\infty}$ satisfy the differential equation defined by (3), (14), and (15).

From (15), we easily see that, for $\alpha \neq 0,1,2, \ldots$,

$$
\operatorname{degree}\left[a_{i}(x)\right]=i, \quad i=1,2,3, \ldots
$$


This implies that if $N>0$ the differential equation is of infinite order in that case.

For nonnegative integer values of $\alpha$, we have

$$
\begin{cases}\text { degree }\left[a_{i}(x)\right]=i, & i=1,2, \ldots, \alpha+2, \\ \operatorname{degree}\left[a_{i}(x)\right]=\alpha+2, & i=\alpha+3, \alpha+4, \ldots, 2 \alpha+4, \\ a_{i}(x)=0, & i>2 \alpha+4\end{cases}
$$

This implies that for nonnegative integer values of $\alpha$ and $N>0$ the differential equation is of order $2 \alpha+4$.

\section{REFERENCES}

1. R. Koekoek, Koornwinder's Laguerre polynomials, Delft Progress Rep. 12 (1988), 393-404.

2. T. H. Koornwinder, Orthogonal polynomials with weight function $(1-x)^{\alpha}(1+x)^{\beta}+$ $M \delta(x+1)+N \delta(x-1)$, Canad. Math. Bull. 27 (1984), 205-214.

3. A. M. Krall, Orthogonal polynomials satisfying fourth order differential equations, Proc. Roy. Soc. Edinburgh Sect. A 87 (1981), 271-288.

4. A. M. Krall and L. L. Littlejohn, On the classification of differential equations having orthogonal polynomial solutions. II, Ann. Mat. Pura Appl. 149 (1987), 77-102.

5. H. L. Krall, Certain differential equations for Tchebycheff polynomials, Duke Math. J. 4 (1938), 705-718.

6. __ On orthogonal polynomials satisfying a certain fourth order differential equation, The Pennsylvania State College Studies, No. 6, 1940.

7. L. L. Littlejohn, On the classification of differential equations having orthogonal polynomial solutions, Ann. Mat. Pura Appl. 93 (1984), 35-53.

8. __ An application of a new theorem on orthogonal polynomials and differential equations, Quaestiones Math. 10 (1986), 49-61.

9. L. L. Littlejohn and A. M. Krall, Orthogonal polynomials and singular Sturm-Liouville systems. I, Rocky Mountain J. Math. 16 (1986), 435-479.

Menelaoslaan 4, 5631 LN Eindhoven, The Netherlands

Faculty of Technical Mathematics and Informatics, Delft University of Technology, P. O. BoX 356, 2600 AJ Delft, The Netherlands 